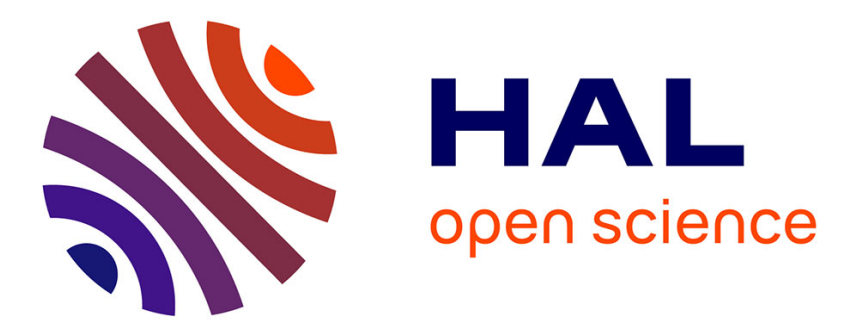

\title{
Modeling \& simulation framework for the inclusion of simulation objectives by abstraction
} Sangeeth Saagar Ponnusamy, Vincent Albert, Patrice Thebault

\section{To cite this version:}

Sangeeth Saagar Ponnusamy, Vincent Albert, Patrice Thebault. Modeling \& simulation framework for the inclusion of simulation objectives by abstraction. International Conference on Simulation and Modeling Methodologies, Technologies and Applications ( SIMULTECH 2014), Aug 2014, Vienne, Austria. pp.385-394. hal-01912545

\section{HAL Id: hal-01912545 \\ https://hal.laas.fr/hal-01912545}

Submitted on 5 Nov 2018

HAL is a multi-disciplinary open access archive for the deposit and dissemination of scientific research documents, whether they are published or not. The documents may come from teaching and research institutions in France or abroad, or from public or private research centers.
L'archive ouverte pluridisciplinaire HAL, est destinée au dépôt et à la diffusion de documents scientifiques de niveau recherche, publiés ou non, émanant des établissements d'enseignement et de recherche français ou étrangers, des laboratoires publics ou privés. 


\title{
MODELING \& SIMULATION FRAMEWORK FOR THE INCLUSION OF SIMULATION OBJECTIVES BY ABSTRACTION
}

\author{
Sangeeth saagar Ponnusamy ${ }^{1 \& 2}$, Vincent Albert ${ }^{2}$ and Patrice Thebault ${ }^{1}$ \\ ${ }^{1}$ Airbus Operations SAS, 316 Route de Bayonne, 31060, Toulouse, France \\ ${ }^{2}$ LAAS-CNRS, 7 Avenue du Colonel Roche, F-31077, Toulouse, France \\ \{sangeeth-saagar.ponnusamy,patrice.thebault\}@airbus.com,valbert@laas.fr
}

Keywords: Abstraction, Compatibility, Experimental Frame, Formal Matching, Hybrid Systems, Simulation

\begin{abstract}
Abstractions of experimental frame components with respect to simulation objectives are discussed with a hybrid system simulation application. Validity assessment through behavioural compatibility criteria described by the trace inclusion framework is given. The simulation objectives are associated with modelling abstractions by such a framework and described in established modeling \& simulation framework. Consistent abstractions from hierarchically ordered posets for stimulant and observer models in experimental frame are discussed. A landing gear example is taken and testability through primary experimental frame component abstractions was observed for the given simulation requirements. The formal framework under development is briefly discussed at the end in the context of applicability and derivability of experimental frame and fidelity of simulation.
\end{abstract}

\section{INTRODUCTION}

A system maps input signals to output signals with an underlying dynamic. Hybrid dynamics in systems arise out of interaction between continuous dynamics and discrete dynamics and are found in a myriad of real world systems, both complex and simple alike. Modeling and Simulation (M\&S) of such dynamics is difficult due to mutual interaction of discrete jumps and continuous flows. Abstractions are always employed in modeling systems and this is more so true in hybrid systems, however, in modeling such a system, the choice of abstractions are crucial to reach the objective of simulation. However, an abstraction is valid only for a given validation objective and in [Albert, 2009] validity of simulation is discussed in terms of class of abstractions. Abstractions of hybrid systems especially for safety verification were widely discussed in [Girard, 2007]. Abstraction must capture the dual relationship between the model and its intended purpose.

This paper describes the formal approach to reach this intended objective of simulation through the abstraction of Experimental Frame (EF) components described in the established M\&S framework by Zeigler [Zeigler, 1984]. The approach is illustrated with an application to EF abstraction refinement and verification of a hybrid system simulation.

\section{MODELING ABSTRACTIONS}

A model is always an abstraction of reality and in modeling and simulation of complex systems, often the difficulty is finding and using consistent and valid abstractions to model the simulated real world system with respect to simulation requirement. In the context of increased usage of simulation as a means to design and analyse real world complex systems, a Model-Based Systems Engineering approach is important in development and usage of simulation products. This is more so true in developing a complex simulation product where the component models are developed by different stakeholders and a common frame of reference must exist in terms of implementing consistent abstractions in the experimental frame.

From systems perspective, consistency is evaluated through traceability and verification, whereas validity is evaluated through validation. In a 
simulation framework, abstraction of the systems to simulate the System Under Test (SUT) includes abstraction of the stimulant and environmental systems and this paper deals with the consistency and validity of stimulant abstractions.

\subsection{Experimental Frame}

In the context of studying a system through simulation, the concept of experimental frame introduced in [Zeigler, 1984] is used to describe experimental scenarios under which the system and corresponding models will be used. An EF defines controllability and observability means to stimulate and observe the model temporal evolution.

The systems approach in segregating SUT and $\mathrm{EF}$ is often the case in reality when system development and its validation through simulation are done by two different entities. The language and its level of abstraction need to be coherent to derive any meaningful conclusion from the simulation results about the real system.

An Experimental Frame, in general, is composed of a Generator (G), Transducer (T) and Acceptor (A). A classical illustration of EF as depicted in [Zeigler, 1984] is shown in the following figure.

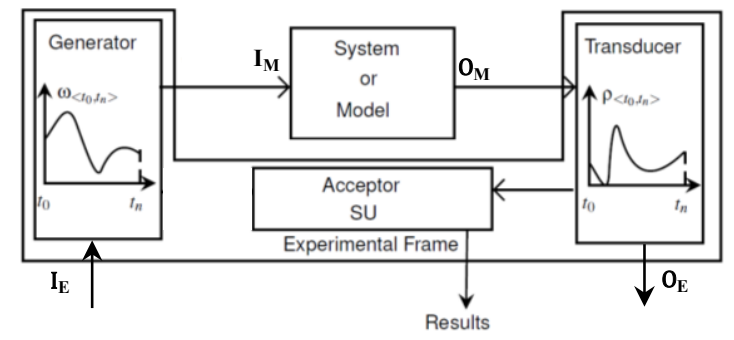

Figure 1: Experimental Frame.

Traoré et al defines an EF in the form of following tuple in [Traoré, 2010]

$$
\mathrm{EF}=<\mathrm{T}, \mathrm{I}_{\mathrm{M}}, \mathrm{I}_{\mathrm{E}}, \mathrm{O}_{\mathrm{M}}, \mathrm{O}_{\mathrm{E}}, \Omega_{\mathrm{M}}, \Omega_{\mathrm{E}}, \Omega_{\mathrm{C}}, \mathrm{SU}>
$$

where $\Omega_{\mathrm{E}} \subseteq\left(\mathrm{T}, \mathrm{I}_{\mathrm{E}}\right), \Omega_{\mathrm{C}} \subseteq\left(\mathrm{T}, \mathrm{O}_{\mathrm{M}}\right)$ and $\Omega_{\mathrm{M}} \subseteq\left(\mathrm{T}, \mathrm{I}_{\mathrm{M}}\right)$ with $\mathrm{T}$ is the time base $\mathrm{I}_{\mathrm{M}, \mathrm{E}}$ are the input variable of model and $\mathrm{EF}$ $\mathrm{O}_{\mathrm{M}, \mathrm{E}}$ are the output variable of model and $\mathrm{EF}$ $\Omega_{\mathrm{M}}$ are the set of segments injected onto the model inputs

$\Omega_{\mathrm{E}}$ are the set of admissible input segments for the experimental control

$\Omega_{\mathrm{C}}$ are the set of segments observed onto the model outputs
SU is the set of conditions, also referred to as summary mappings establishing relationship between inputs and outputs within a frame.

The acceptor dictates the acceptance conditions for simulation and encoded in temporal logic

$$
\Omega_{\mathrm{C}} \subseteq\left(\mathrm{T}, \mathrm{O}_{\mathrm{M}}\right) \vDash\left\{\varphi_{1}, \varphi_{2} \ldots \varphi_{\mathrm{n}}\right\}
$$

where $\varphi_{i=1 . . n}$ are the requirements defined in a formalism such as temporal logic. An example could be, response time for the steady state should be below a given time limit, $\varphi_{\mathrm{i}}=\mathrm{t}_{\text {sst }}<\mathrm{t}_{\text {limit }}$.

The generator acts as an input stimulus for the model, whose outputs are transformed by a transducer into a comprehensible form, which are in turn compared against a set of acceptable conditions specified by an acceptor. In addition, the EF may contain environmental models which simulate the real environment in which the SUT operates. Thus the EF components may be classified broadly as primary and secondary components with the former being the prime drivers of simulation, namely generator, acceptor and transducer, and the latter being environmental models. The components could be interconnected and hierarchically composed to build an EF. However, it must be noted that abstraction of environmental models are equally important as such models are seldom absent in an EF.

\subsubsection{EF Applicability \& Derivability}

In [Albert, 2010], the concepts of homomorphism, applicability and derivability are discussed in the framework of M\&S. A morphism relation establishes correspondence between a concrete model and an abstract model and such a relation between two models is called homomorphism when the transition and output function has been preserved i.e. behavioural equivalence. Applicability and derivability are more structural concepts in that the former determines whether an EF can be applied to a model and the later determines the extent of such an application. Applicability and derivability defines compatibility criteria between a model and EF. This compatibility is influenced by the abstraction level of $\mathrm{EF}$ components and the paper deals with the applicability of such an abstraction. Figure 2, taken from [Zeigler, 1984], illustrates this concept between the derivability and hierarchy of abstractions and the relation between them through applicability. 


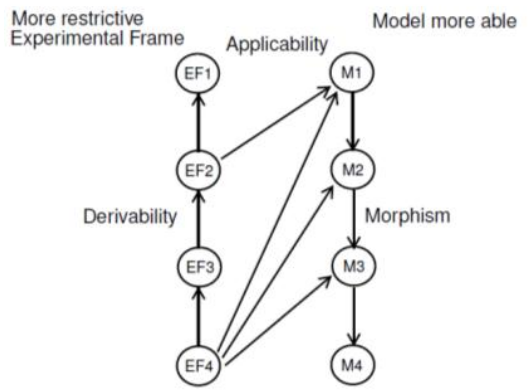

Figure 2: Morphism, Applicability \& Derivability relations.

\subsection{Validity in Experimental Frame}

In general, a model is said to be valid if it satisfies the experimental frame. In this context, in [Albert, 2009], experimental frames were proposed in terms of model usage domain and objective domain called Simulation Domain of Use (SDU) and Simulation Objective of Use (SOU) respectively.

Simulation validity in other words can be defined as the compatibility between SDU and SOU. Compatibility, in general, is defined as the degree of conformity between the considered entities. A valid simulation has the prerequisite of syntactic and semantic compatibility between the SDU and SOU. A study on semantic compatibility between the ports of simulation models based on ontologies was done in [Man, 2009]. Similarly, in [Albert, 2010], compatibility between EF and model interfaces were discussed in terms of syntactics parameters such as topology, scope, type signature, I/O relation. In this paper, however, compatibility is discussed in terms of validity through abstraction. In simulating a complex system which is hierarchically composed of different subsystems, modeling abstraction choices in building a SDU consistent with the simulation objectives described by SOU will yield this compatibility. In addition, simulation product validity is an aggregation of the problem of correctness and validity. The correctness of implementation or verification is not discussed here and only the abstraction influence of primary EF on validity is discussed.

\subsubsection{Primary EF Component Validity}

SDU and SOU intuitively refers to model behavioural limits and model behavioural expectations respectively. Then the key question is how to drive the model behaviour to reach its intended expectations in the context of simulation. In other words, what are the necessary and consistent abstractions to be made in the EF components to drive the SUT to an acceptable degree of validity? This paper deals with the reachability of SOU through primary EF component abstractions. The reachability of SOU through environmental model abstractions and their composition with primary components are subject of another study and are not discussed here.

From the systems perspective, testability of a system is based on the controllability and observability of the system components. Controllability and observability defines the ease of bringing and propagating data to the input and output of the component respectively. Thus the abstraction of primary EF components must result in adequate testability conditions with respect to the simulation objectives.

In [Foures, 2013], a method of defining the intended purpose of simulation for discrete event simulation of a continuous system was presented by Damien et al. In [Foures, 2012], a formal compatibility between EF and FD-DEVS model was proposed in terms of metrics defined on scope, precision and state space. The state space metric was discussed in terms of trace inclusion and a truth table was proposed to describe the model coverage by EF. This study is an extension of such definition of SDU and SOU to simulation of a hybrid system in the context of input abstraction and its subsequent compatibility to an EF.

The compatibility is discussed in terms of reachability of the SUT where reachability is defined as the set of all possible states reachable by a system and is used to verify temporal logic properties defined as safety etc. In this context of definition of validation requirements, it is important to distinguish between simulation validity and system validity. Simulation validity answers whether the simulation is adequate to answer questions on system validation. System validation is validation of system with respect to its requirements. Simulation validity is a prerequisite of system validity and thus decisions taken at any stage along the $\mathrm{V}$ cycle where simulation is used as a means of Verification \& Validation, it is intrinsically tied to the key question of simulation fidelity. A system is said to be valid by simulation only when the simulation itself is valid and thus it is a necessary and sufficient condition for system validity assessment through simulation. Let $\varphi_{\text {sys }}$ and $\varphi_{\text {sim }}$ be system and simulation requirements respectively on the system $\left(\mathrm{S}_{s y s}\right)$ and its representation $\left(\mathrm{S}_{\text {sim }}\right)$.

System validation through simulation implies the acceptor input i.e. model output, satisfies system requirements and thereby simulation requirements, 


$$
\Omega_{C} \vDash \varphi_{\text {sys }} \Rightarrow \vDash \varphi_{\text {sim }}
$$

where, $\Omega_{C} \vDash \varphi_{\text {sim }}$ means simulation validity. The converse may not be true $\Omega_{C} \vDash \varphi_{\text {sim }} \nvdash \vDash \varphi_{\text {sys }}$.

The system validity assessment by simulation thus becomes

$$
\varphi_{\mathrm{i}=1 . . \mathrm{n}}=\varphi_{\text {sys }} \mathrm{U} \varphi_{\text {sim }}
$$

In other words, the above equation dictates that reachability under input stimuli $\left(\Omega_{\mathrm{M}}\right)$ to the model from $\mathrm{EF}\left(\Omega_{\mathrm{E}}\right)$ must result in model output $\left(\Omega_{\mathrm{C}}\right)$ satisfying $\varphi_{i=1 . . n}$ to be a valid model.

It may be recalled that the distance between system and simulation validation is introduced by abstraction of the system as a simulation model. The study deals with what are the necessary and consistent modeling abstractions to be implemented in simulation such that they are consistent with system validation requirements. In other words, choosing abstractions such that the simulation is adequate i.e. valid to draw any meaningful conclusion about the real system. Assuming correct environmental model abstraction, the question is abstraction of the primary EF components in driving the simulation to its objective with respect to SDU. In this paper, through reachability of SUT, necessary and consistent primary EF abstractions with respect to system requirements are discussed.

\subsubsection{Primary EF Component Abstraction}

The primary components of EF are given as

$$
\mathrm{M}_{\mathrm{p}}=<\mathrm{T}, \mathrm{X}_{\mathrm{p}}, \mathrm{Y}_{\mathrm{p}}>\mid \mathrm{p}=\{\mathrm{G}, \mathrm{T}, \mathrm{A}\}
$$

where $\mathrm{X}$ and $\mathrm{Y}$ are input and output variables defined with over a time base T. Similar to the general EF definition, we define $\Omega_{\mathrm{Y}_{\mathrm{p}}} \subseteq\left(\mathrm{T}, \mathrm{Y}_{\mathrm{p}}\right)$, are the set of output segments $\Omega_{\mathrm{X}_{\mathrm{p}}} \subseteq\left(\mathrm{T}, \mathrm{X}_{\mathrm{p}}\right)$, are the set of input segments

A morphism relation establishes correspondence between a concrete model and its abstract version through abstraction operation. Abstractions are manyfold depending on the simulation objectives and hypotheses. From the classes of abstractions defined in [Albert, 2009], we define abstraction operation as $\alpha$ over an abstraction class. Such abstractions are related by binary relations forming a partial order. A partially ordered set or a poset is a set $\mathrm{P}=(\leqslant, \mathrm{S})$ with reflexive, transitive relation on a set $\mathrm{S}$. The hierarchy of abstractions could be defined as a partial order relation over a finite lattice.

$$
\mathrm{M}_{\mathrm{p}}^{\mathrm{C}} \stackrel{\alpha_{\mathrm{i}}^{1}}{\rightarrow} \mathrm{M}_{\mathrm{p}}^{1} \stackrel{\alpha_{\mathrm{i}}^{2}}{\rightarrow} \ldots \mathrm{M}_{\mathrm{p}}^{\mathrm{n}}
$$

Different abstraction operations may be feasible over such a finite lattice whose height is defined by a set $\mathrm{N}$. The valid set of abstractions among them are defined by

$$
\left\{\alpha_{\mathrm{p}}^{\mathrm{n}}\right\} \vDash\left\{\varphi_{1}, \varphi_{2} \ldots \varphi_{\mathrm{n}}\right\} \mid \mathrm{n} \in \mathrm{N}
$$

In addition to abstraction of model semantics, model interfaces are abstracted based on their syntax definition and semantics it handles. The syntactics (number of ports, coupling, structure) and semantics (data type, type signature) of EF and SOU interfaces must be compatible and are defined in terms of a partial order relation. Such a definition followed by an inclusion criterion will help address the simulation validity with respect to abstractions.

The general inclusion relation between the admissible model input segments with respect to its capabilities are defined by

$$
\Omega_{\mathrm{MSOU}} \subseteq \Omega_{\mathrm{M}}
$$

It must be noted that there could be interconnection $(\mathrm{i} \rightarrow \mathrm{d})$ between environmental models and primary components and the applicability extends to them as well. In EF definition as a tuple in [Albert, 2010], the coupling between models $\mathrm{M}$ with identifiers $\mathrm{I}$ is given by $\mathrm{Z}$.

$$
\mathrm{EF}=<\mathrm{T}, \mathrm{X}_{\mathrm{EF}}, \mathrm{Y}_{\mathrm{EF}},\left\{\mathrm{M}_{\mathrm{d}}\right\},\left\{\mathrm{I}_{\mathrm{d}}\right\}, \mathrm{Z}_{\mathrm{EF}_{\mathrm{i} \rightarrow \mathrm{d}}}>
$$

where $\mathrm{EF}=\{\mathrm{SOU}, \mathrm{SDU}\}$

The experimental control segments to model, $\Omega_{\mathrm{E}}$ and acceptor input, $\Omega_{\mathrm{C}}$ then becomes

$$
\begin{aligned}
\Omega_{\mathrm{M}} & =\Omega_{\mathrm{Y}_{\mathrm{G}}} \cup \Omega_{\mathrm{Y}_{\mathrm{EM} \rightarrow \mathrm{M}}} \\
\Omega_{\mathrm{C}} & =\Omega_{\mathrm{X}_{\mathrm{T}}} \cup \Omega_{\mathrm{X}_{\mathrm{M} \rightarrow \mathrm{EM}}}
\end{aligned}
$$

Acceptance conditions require transduced outputs or outputs of the SUT or environmental models. The compatibility is given by

$$
\Omega_{\mathrm{Y}_{\mathrm{T}}} \vee \Omega_{\mathrm{Y}_{\mathrm{M}}} \vee \Omega_{\mathrm{Y}_{\mathrm{EM} \rightarrow \mathrm{A}}} \subseteq \Omega_{\mathrm{X}_{\mathrm{A}}}
$$

Utilising such definition, applicability is extended as 


$$
\begin{aligned}
& \Omega_{\mathrm{Y}_{\mathrm{G}}} \cup \Omega_{\mathrm{Y}_{\mathrm{EM} \rightarrow \mathrm{M}}} \subseteq \Omega_{\mathrm{X}_{\mathrm{M}}} \\
& \Omega_{\mathrm{Y}_{\mathrm{T}}} \cup \Omega_{\mathrm{X}_{\mathrm{M} \rightarrow \mathrm{EM}}} \subseteq \Omega_{\mathrm{Y}_{\mathrm{M}}}
\end{aligned}
$$

The compatibility criteria described above also includes model constraints, $\emptyset_{\mathrm{M}}$ defined by the behavioural limits in terms of possible reachable states (S), in other words SDU, as well as the constraints on the inputs (X) and outputs (Y).

$$
\emptyset_{\mathrm{M}}=\emptyset\left\{\mathrm{X}_{\mathrm{M}}, \mathrm{Y}_{\mathrm{M}}, \mathrm{S}_{\mathrm{M}}\right\}
$$

Constraints on state, output and input are defined for all the EF and SUT and violation of such constraints results in inconsistency. The constraint on the state evolution is given below

$$
\begin{aligned}
& \forall \mathrm{S}_{\mathrm{M}}^{\mathrm{i}} \stackrel{\tau}{\rightarrow} \mathrm{S}_{\mathrm{M}}^{\mathrm{i}+1} \text { such that } \\
& \left(\mathrm{S}_{\mathrm{M}}^{\mathrm{i}}, \mathrm{S}_{\mathrm{M}}^{\mathrm{i}+1}\right) \in \emptyset_{\mathrm{M}}\left(\mathrm{S}_{\mathrm{M}}\right)
\end{aligned}
$$

Intuitively, the above equation lays out a consistency criteria such that the under transition relation, $\tau$, the evolution of state from step $i$ to $i+1$ respect the constraints imposed on the state space. Similarly, such definition can be extended to inputs and outputs.

\subsection{Model Coverage Metric}

The compatibility state space metric defined by trace inclusion is used to analyse the extent of model coverage by primary EF components and thereby quantify the abstraction with respect to simulation objectives. In this context, four criteria have been proposed with respect to this model coverage metric,

Valid : EF Abstractions are consistent with simulation objectives.

$$
\left\{\Omega_{\mathrm{Y}_{\mathrm{M}}} \mid \vDash \varphi_{\mathrm{i}} \wedge\left\{\mathrm{X}_{\mathrm{M}}, \mathrm{Y}_{\mathrm{M}}, \mathrm{S}_{\mathrm{M}}\right\} \in \emptyset_{\mathrm{M}}\right\}
$$

Partially Valid: EF Abstractions are partially consistent with simulation objectives.

$$
\begin{gathered}
\left\{\Omega_{\mathrm{Y}_{\mathrm{M}}}^{1} \in \Omega_{\mathrm{Y}_{\mathrm{M}}} \mid \vDash \varphi_{\mathrm{i}} \wedge\right. \\
\left.\left\{\mathrm{X}_{\mathrm{M}}, \mathrm{Y}_{\mathrm{M}}, \mathrm{S}_{\mathrm{M}}\right\} \in \emptyset_{\mathrm{M}}\right\} \\
\left\{\Omega_{\mathrm{Y}_{\mathrm{M}}}^{2} \in \Omega_{\mathrm{Y}_{\mathrm{M}}} \mid \not \varphi_{\mathrm{i}} \wedge\right. \\
\left.\left\{\mathrm{X}_{\mathrm{M}}, \mathrm{Y}_{\mathrm{M}}, \mathrm{S}_{\mathrm{M}}\right\} \in \emptyset_{\mathrm{M}}\right\}
\end{gathered}
$$

Properties $\varphi_{i}$ belonging to the same class could be hierarchical from high level to low level and are validated sequentially $\quad\left(\Omega_{\mathrm{Y}_{\mathrm{M}}} \vDash \varphi_{\mathrm{i}=\mathrm{n}} \Rightarrow \Omega_{\mathrm{Y}_{\mathrm{M}}} \vDash\right.$ $\left.\varphi_{\mathrm{i}=\mathrm{n}+1}\right)$.
Invalid : EF abstractions are not consistent with simulation objectives and resulting model behaviour violates the requirements

$$
\left\{\Omega_{\mathrm{Y}_{\mathrm{M}}} \mid \not \varphi_{\mathrm{i}} \wedge\left\{\mathrm{X}_{\mathrm{M}}, \mathrm{Y}_{\mathrm{M}}, \mathrm{S}_{\mathrm{M}}\right\} \in \emptyset_{\mathrm{M}}\right\}
$$

Incompatible : EF abstractions are not consistent with simulation objectives and the resulting model behaviour violates the constraints.

$$
\left.\left\{\Omega_{\mathrm{Y}_{\mathrm{M}}}|\not| \mathrm{X}_{\mathrm{M}}, \mathrm{Y}_{\mathrm{M}}, \mathrm{S}_{\mathrm{M}}\right\} \in \emptyset_{\mathrm{M}}\right\}
$$

The EF abstraction is said to be valid if the resulting reachable states are achievable and covered. The abstractions of primary EF components resulting in such validity are denoted by $\alpha_{\mathrm{p}}$, where $\mathrm{p}=\{\mathrm{G}, \mathrm{T}, \mathrm{A}\}$.

In the primary EF model abstractions, certain abstractions are used to drive the simulation to its objective and are called design abstractions $\alpha_{\mathrm{p}}^{\mathrm{d}} \in \alpha_{\mathrm{p}}$. For example the generator abstraction, $\alpha_{\mathrm{G}}^{\mathrm{d}} \in \alpha_{\mathrm{G}}$ resulting in SUT input $\Omega_{\mathrm{M}}^{\mathrm{d}} \in \Omega_{\mathrm{M}}$ driving the simulation output is given by notation $\alpha_{\mathrm{G}}^{\mathrm{d}} \mapsto \Omega_{\mathrm{M}}^{\mathrm{d}}$. More details can be found with an example in the following application case.

\section{APPLICATION CASE}

As an example application for our approach, verification of behavioural properties of an aircraft landing gear described in [Boniol, 2014] was taken. An aircraft landing gear is used to support the weight of the aircraft during landing and ground operations. The conventional retractable landing gear is tricycle type with two aft gears and one front gear attached to the main structure of aircraft. In the following example, other details of the landing gear system such as brakes, retractable mechanism, warning devices, fairing, cowling, structures and other auxiliary systems are not discussed.

\subsection{Problem Formulation}

The landing gear is extended or retracted by a set of hydraulic actuators and the system is controlled digitally in normal mode and analogically in emergency mode. The SUT is the landing gear digital control logic which controls the opening or closing of flow control valves to the actuators. In normal operation, upon the extend command, the doors are opened and the landing gear is extended and upon retract command, the gear is retracted followed by door closure. The opening and closing of doors are not simulated in this case. The general 
architecture of landing gear is given below with the presence of a single actuator and could be extended to the full system of all the landing gears,

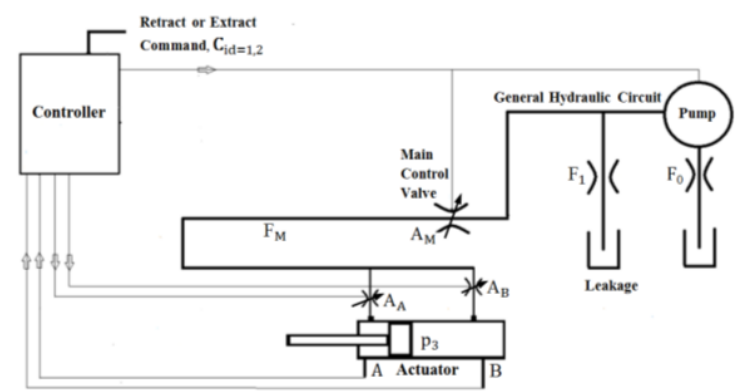

Figure 3: Landing gear

The architecture of the hydraulic part is described in Figure 3 and only the principles of the motion mechanism are discussed. The landing gear motion is performed by a set of actuating cylinders. The cylinder piston position corresponds to the landing gear position and for each landing gear, a cylinder retracts or extends it. Hydraulic power is provided to the cylinders by a set of electro-valves, where one main electro-valve supplies the specific electro-valves for closing or opening with hydraulic power from the aircraft hydraulic circuit. The hydraulic power is supplied to the landing gear circuit by a pump with flow Q. The actuator part of the model is inspired from a MATLAB example of the single hydraulic cylinder simulation [MATLAB, 2014]. The architecture of the actuator cylinder is same except for the presence of two openings at the ends of actuator cylinder marked A and B denoting retracted and extended positions respectively.

The working mechanism is briefly given as follows, initially the control logic receives the pilot command to extend or retract and, activates the pump. As the flow from pump is passed through the opening main control valve orifice with area $A_{M}$, the pressure, $\mathrm{p}_{3}$ starts building at the end $\mathrm{A}$ or $\mathrm{B}$, depending on the pilot input $\mathrm{C}_{1}$, to extend or $\mathrm{C}_{2}$, to retract the gear. Once the pressure differential exceeds a certain threshold, $\mathrm{K}_{p}^{A \vee B}$, the piston starts moving until it reaches the other end or chamber pressure equalizes the pump pressure, whichever is earlier. Modeling abstractions such as flow coefficients $\left(\mathrm{F}_{0}, \mathrm{~F}_{1} \& \mathrm{~F}_{\mathrm{M}}\right)$, leakage phenomenon, orifice model are kept the same as described in the example for the sake of simplicity. Similarly, the dynamic effect of aerodynamic or ground reactions is not considered and interaction with other aircraft systems is also not considered.

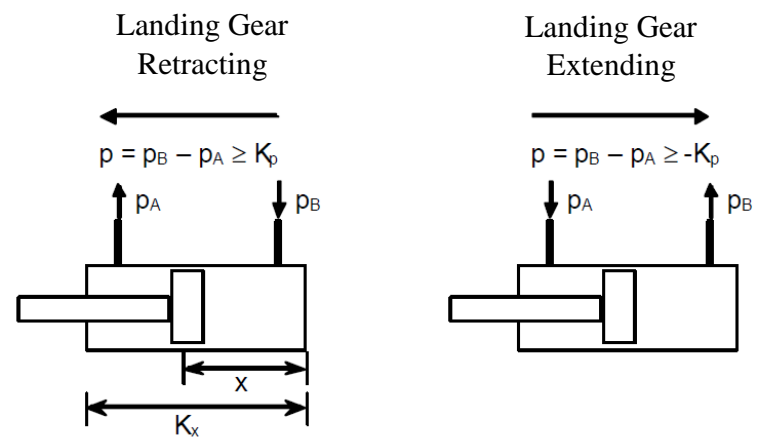

Figure 4: Actuator model [Boniol, 2014]

The inertial differential pressure at the ends $\mathrm{A}$ and $\mathrm{B}$ are $\mathrm{K}_{\mathrm{p}}^{\mathrm{A}}$ and $\mathrm{K}_{\mathrm{p}}^{\mathrm{B}}$ and the dwell time when pressure is below these limits corresponds to the unlock time from the current mode. The length of cylinder is given by $K_{x}$.

\subsection{System Dynamics \& Simulation}

The SUT is modelled as a Finite State Machine (FSM) abstraction, a data type state aggregation abstraction with hypothesis being the system dynamics has four different modes depending on the pilot input and actuator response.

Retracted : The piston is at position A and the differential pressure is below the threshold.

Extending : The modulus of differential pressure is above the threshold, $\mathrm{K}_{\mathrm{p}}^{\mathrm{A}}$, and the piston starts moving from $\mathrm{A}$.

Extended : The piston is at position B

Retracting : The modulus of differential pressure is above the threshold, $\mathrm{K}_{\mathrm{p}}^{\mathrm{B}}$, and the piston starts moving from $B$.

The system remains at the retracted or extended position indefinitely until pilot command has been initiated or failure of hydraulic circuit or both.

The system is modeled in SIMULINK and Stateflow, a widely used commercial tool in modeling and simulation of complex reactive systems based on the finite state machine described by events and actions. Simulations are carried out using a variable step ODE45 solver. Alternatively, such a hybrid system could be modeled in DEVS formalism and solved using QSS algorithms which are more amenable to hybrid system simulation as the state events can be handled much more efficiently by state-quantization algorithms than by time-slicing algorithms.

The SUT is the control logic with the environmental models being that of actuator, pump 
and main control valve. The generator given below supplies the input to generate input segments, $\Omega_{\mathrm{M}}$ of pump flow and main control valve profile apart from pilot commands

The switching modes are illustrated in the following figure.

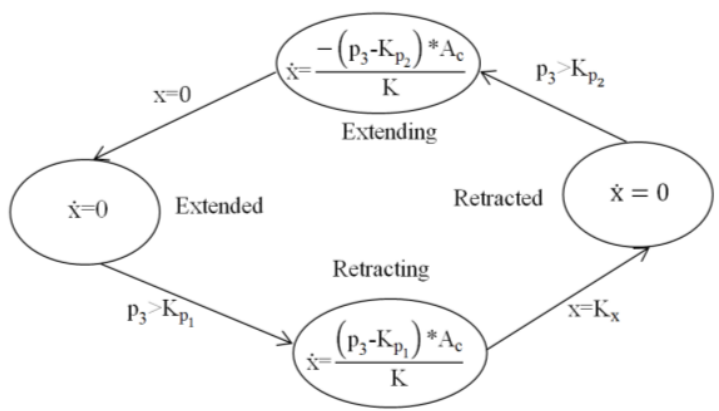

Figure 5: Landing gear hybrid system

\subsection{Simulation Requirements}

Broadly, the requirements are classified as normal and failure modes and the requirements related to normal mode gear function alone listed in [Boniol, 2014] are taken for validity assessment.

The high level SOU functional objective is reaching the mode of operation for the given command. The system should start from extended mode and reach retracted mode when retract command is given and vice versa for extend command.

The other SOU is defined as the data class type abstraction with validity criteria being error tolerance on the maximum time of extending and retracting denoted by $t_{\text {extend }}$ and $t_{\text {retract }}$ respectively.

$$
\begin{aligned}
\varphi_{1}:= & \left\{\forall \mathrm{C}_{1} \mid \mathrm{t}_{1}<\mathrm{t}_{2}<\mathrm{t}_{\text {extend }}\right\} \mathrm{V} \\
& \left\{\forall \mathrm{C}_{2} \mid \mathrm{t}_{3}<\mathrm{t}_{4}<\mathrm{t}_{\text {retract }}\right\}
\end{aligned}
$$

where, $\mathrm{t}_{1}$ is the unlock time (when $\mathrm{p}_{3}<\mathrm{K}_{p}^{A}$ ), $\mathrm{t}_{2}$ is the time of extension, $t_{3}$ is the unlock time (when $\left.\mathrm{p}_{3}<\mathrm{K}_{p}^{B}\right)$ and $\mathrm{t}_{4}$ is the time of retraction. The simulation requirement given in the form of temporal profile with gear angle being measured from horizontal plane is shown in figure 6 .

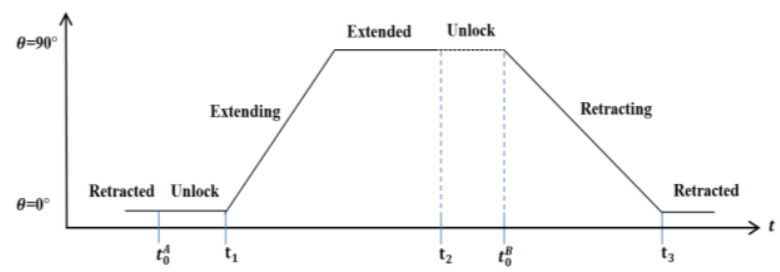

Figure 6: Landing gear output requirement
The state constraints are given as

$$
\emptyset_{\mathrm{M}}=\left\{\mathrm{p}_{3} \in\left[0 \mathrm{p}_{\mathrm{lim}}\right]\right\} \cup\left\{\mathrm{x} \in\left[0 \mathrm{~K}_{\mathrm{x}}\right]\right\}
$$

The simulation is valid if it satisfies the functional and temporal requirements without violating constraints.

\subsection{Experimental Frame}

The specification of the experimental frame defined in Eq 1 is given as follows.

$$
\mathrm{T}=\mathbb{R}
$$

The input and output of the EF are

$$
\begin{gathered}
\mathrm{I}_{\mathrm{E}}=\{\text { start/stop }\} \\
\mathrm{O}_{\mathrm{E}}=\{\Theta\}
\end{gathered}
$$

The input and output of the SUT are

$$
\begin{gathered}
\mathrm{I}_{\mathrm{M}}=\mathrm{Y}_{\mathrm{G} \rightarrow \mathrm{M}} \cup \mathrm{Y}_{\mathrm{EM} \rightarrow \mathrm{M}}=\left\{\mathrm{x}, \mathrm{p}_{3}, \mathrm{C}_{1}, \mathrm{C}_{2}\right\} \\
\mathrm{O}_{\mathrm{M}}=\mathrm{Y}_{\mathrm{M} \rightarrow \mathrm{G}} \cup \mathrm{Y}_{\mathrm{M} \rightarrow \mathrm{EM}}=\left\{\mathrm{A}_{\text {extend }}^{\mathrm{m}}, \mathrm{A}_{\text {retract }}^{\mathrm{n}}, \mathrm{S}_{\mathrm{i}}\right\}
\end{gathered}
$$

where

$\mathrm{i} \rightarrow \mathrm{d}$ gives the interconnection relation

$(\mathrm{m}, \mathrm{n})=(\mathrm{A}, \mathrm{B}) \mathrm{V}(\mathrm{B}, \mathrm{A})$ is the retracting or extending valves

$\mathrm{id}=\{$ retracted, retracting, extended, extending $\}$ are the states describing the phase of the simulation.

The input segments of the EF and model are given based on Eq 1. The acceptor segments are given by

$$
\Omega_{\mathrm{C}}=\left\{\left(\mathrm{S}_{\mathrm{id}}, \mathrm{t}\right),\left(\mathrm{Y}_{\mathrm{M} \rightarrow \mathrm{EM}}, \mathrm{t}\right)\right\}
$$

The environmental abstractions are assumed to be ideal with respect to simulation requirements and only the primary EF components abstractions are discussed in the following section.

The experimental frame is illustrated in figure 7.

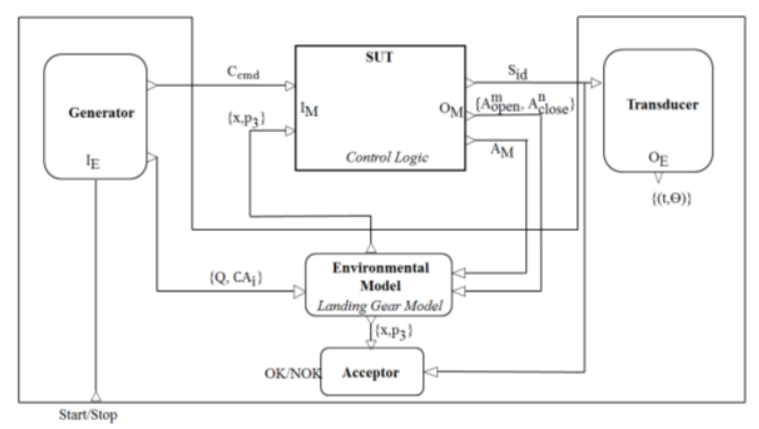

Figure 7: Landing gear hybrid system 
The interconnection between EF and model components can be seen and such a definition helps in coherent model development with respect to simulation objectives.

\subsubsection{Primary EF Abstractions}

The generator, acceptor and transducer are described below.

Generator, G: The input stimulii are the pilot command to retract or extend the gear, pump flow parameters of the main control valve orifice area and pump flow profile.

$$
\mathrm{M}_{\mathrm{G}}=<\mathrm{T}, \mathrm{X}_{\mathrm{G}}, \mathrm{Y}_{\mathrm{G}}>
$$

where, $X_{\mathrm{G}}=\mathrm{I}_{\mathrm{E}}$,

$\mathrm{Y}_{\mathrm{G}}=\left\{\left\{\mathrm{Q}, \mathrm{C}_{\mathrm{Ai}}, \mathrm{C}_{\mathrm{cmd}}\right\} \mid \mathrm{cmd}=\{\right.$ retract, extend $\left.\}\right\}$ $\mathrm{C}_{\mathrm{Ai}}$ is the main orifice valve opening profile.

The computation class abstraction is employed in the form of a Look Up Table and linear interpolation between data points $\mathrm{d}$ for the pump flow and valve opening profile. The pilot command is abstracted as a simple flag.

$$
\alpha_{G}^{1}\left(M_{G}^{C}\right):=f_{l}(t, d)
$$

$\mathrm{M}_{\mathrm{G}}^{\mathrm{C}}$ is the concrete system specification of pump and main control valve. $f_{l}$ is the linear map between data points and time. The design abstraction, $\alpha_{\mathrm{G}}^{\mathrm{d}}$ defined in section 2.3, in this case is $\mathrm{Q}$ and $\mathrm{C}_{\mathrm{Ai}}$.

Transducer, T: The state of the model is transduced in terms of gear rotation angle. The transducer model is given by

$$
\mathrm{M}_{\mathrm{T}}=<\mathrm{T}, \mathrm{X}_{\mathrm{T}}, \mathrm{Y}_{\mathrm{T}}>
$$

where, $X_{T}=\left\{S_{i d}\right\} Y_{T}=\left\{\Theta \mid O_{E}\right\}$

The transducer is abstracted as

$$
Y_{T}=\left\{\begin{array}{cc}
0 & \text { if } S_{i d}=S_{\text {retract }} \\
f(x) & \text { if } S_{\text {id }}=\left(S_{\text {retracting }} \vee S_{\text {extending }}\right) \\
90 & \text { if } S_{\text {id }}=S_{\text {extend }}
\end{array}\right.
$$

The map could be a simple linear function (eg: $\left.90 *\left(\mathrm{~K}_{\mathrm{x}} / \mathrm{x}\right)\right)$ or may be dependent on velocity, transmission delay etc.

Acceptor, A : The acceptor includes conditions to check physical violation constraints such as negative pressure defined as $\emptyset_{\mathrm{M}}$ and semantics of modes formalized in temporal logic.

$$
\alpha_{A}^{1}\left(M_{A}^{C}\right):=\left(\begin{array}{c}
\square\left(C_{1} \wedge S_{\text {retracted }} \rightarrow \diamond S_{\text {extended }}\right) \vee \\
\square\left(C_{2} \wedge S_{\text {extended }} \rightarrow \diamond S_{\text {retracted }}\right)
\end{array}\right)
$$

where $\mathrm{M}_{\mathrm{A}}^{\mathrm{C}}$ is the concrete acceptance conditions specified in temporal logic formalism. Simulation validity conditions could also be specified in it.

\subsubsection{Results}

A typical retract and extend operation of the landing gear is shown below for a sample simulation
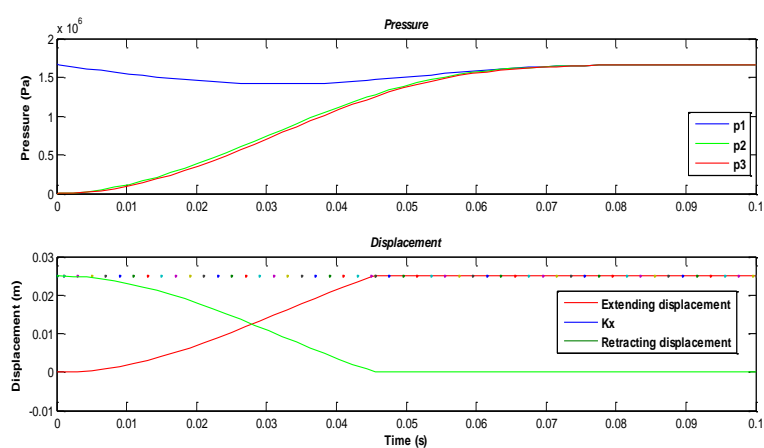

Figure 8: Landing gear output

The fall in pressure at the pump, $\mathrm{p}_{1}$ and subsequent rise in pressure at the main control valve, $\mathrm{p}_{2}$ and downstream in the chamber, $\mathrm{p}_{3}$ are seen. The piston displacement, $\mathrm{x}$ for both extending (red) and retracting (green) until it reaches other end of cylinder is seen. In failure cases such as pump failure, the piston stops before it reaches the other end, which is not shown here. In essence, in normal mode, once the piston reaches the other end, the control logic closes the control valve and pressure equalises in the circuit. For the sake of simplicity the closing of main valve is not simulated.

The method allows to abstract the valid primary $\mathrm{EF}$ components with respect to requirements based on sample simulation runs. In the present simulation the pump flow, $\mathrm{Q}$ and main valve opening, $\mathrm{A}_{\mathrm{M}}$ are the input design parameters, $\mathrm{I}_{\mathrm{M}}^{\mathrm{d}}$. Recalling definition in section 2.3, the generator design abstraction, $\alpha_{\mathrm{G}}^{\mathrm{d}} \in \alpha_{\mathrm{G}}$ resulting in model input, $\Omega_{\mathrm{M}}^{\mathrm{d}} \in \Omega_{\mathrm{M}}$ driving the simulation output is given by notation, $\alpha_{\mathrm{G}}^{\mathrm{d}} \mapsto$ $\Omega_{\mathrm{M}}^{\mathrm{d}}$. Then the trace inclusion criteria allows to classify them as

$$
\begin{aligned}
& \text { Valid : } \\
& \qquad\left\{\alpha_{\mathrm{G}}^{\mathrm{d}} \mapsto \Omega_{\mathrm{M}}^{\mathrm{d}} \mid \vDash \varphi_{1} \wedge\left\{\mathrm{x}, \mathrm{p}_{3}\right\} \in \emptyset_{\mathrm{M}}\right\}
\end{aligned}
$$


Invalid:

$$
\left\{\alpha_{\mathrm{G}}^{\mathrm{d}} \mapsto \Omega_{\mathrm{M}}^{\mathrm{d}} \mid \not \varphi_{1} \wedge\left\{\mathrm{x}, \mathrm{p}_{3}\right\} \in \emptyset_{\mathrm{M}}\right\}
$$

$$
\begin{aligned}
& \text { Partially Valid: } \\
& \left\{\begin{array}{l}
\left\{\alpha_{\mathrm{G}}^{\mathrm{d}} \mapsto \Omega_{\mathrm{M}}^{\mathrm{d} 1} \in \Omega_{\mathrm{M}}^{\mathrm{d}} \mid \vDash \varphi_{1} \wedge\left\{\mathrm{x}, \mathrm{p}_{3}\right\} \in \emptyset_{\mathrm{M}}\right\} \\
\left\{\alpha_{\mathrm{G}}^{\mathrm{d}} \mapsto \Omega_{\mathrm{d}}^{\mathrm{d} 2} \in \Omega_{\mathrm{M}}^{\mathrm{d}} \mid \not \varphi_{1} \wedge\left\{\mathrm{x}, \mathrm{p}_{3}\right\} \in \emptyset_{\mathrm{M}}\right\}
\end{array}\right.
\end{aligned}
$$

Incompatible:

$$
\left.\left\{\alpha_{\mathrm{G}}^{\mathrm{d}} \mapsto \Omega_{\mathrm{M}}^{\mathrm{d}}|\not| x, \mathrm{p}_{3}\right\} \in \emptyset_{\mathrm{M}}\right\}
$$

The pump flow and cross section parameters of main valve are thus classified with respect to simulation objectives. Similar such abstraction for transducer $\alpha_{\mathrm{T}}^{\mathrm{d}} \in \alpha_{\mathrm{T}}$ and acceptor $\alpha_{\mathrm{A}}^{\mathrm{d}} \in \alpha_{\mathrm{A}}$ driving the SUT can be defined respectively, though it is not used in the current study. Such design abstractions can help for example drive the simulation to its objective by observing and monitoring the results. The aggregate effects of all such primary abstractions are observed onto the model output.

It may be noted that the requirements $\varphi_{1}$ defined belong to temporal class in that in certain cases validation of a lower level requirement implicitly validates the higher level requirement. Assuming the acceptor abstraction $\alpha_{A}^{1}$ is given as a requirement $\varphi_{2}$ then validation of temporal behaviour specified as $\varphi_{1}$ implies validation of semantics of mode specified as $\varphi_{2}$.

The abstraction influence of primary EF components on simulation validity can thus be studied using such a validity criteria. Abstraction classification and hierarchical composition implemented in a tool will help in extracting abstractions which are necessary and consistent with simulation objectives. Building a repository of such abstractions with respect to objectives could be used to derive and reuse concepts based on the ontology framework, also based on the lattice concepts. The unified simulation method thus helps in better development of models corresponding to requirements.

\section{FUTURE WORK}

The present study deals with primary EF component abstraction compatibility with SOU. The notions are based on trace inclusion and a formal tool needs to be built to quantify this abstraction. However, notion of reachability is more pertinent than simulation for hybrid systems since an exhaustive breadth first search of state space through reachability analysis, difficult as it might be in terms of computational cost, yields formal verification of system. In this regard, various reachability tools such as MATISSE, UPPAAL, StateEx may be used and the inclusion relation of reachable state space of SDU with respect to SOU could be checked. Problems of scalability of these reachability methods were discussed widely in literature with potential solutions of using abstractions to alleviate the computational burden. The next step would be extending this method of reachability inclusion through formal verification tools.

The influence of modeling abstractions especially of environmental models in EF are not discussed here and quantification of abstraction effect on the model reachability with respect to its objective is of fundamental importance in the usage of simulation as a means of analysis and design of real world systems. A correct 'by design' of abstraction with respect to simulation objectives based on the concepts of approximate bisimulation [Girard, 2007] and Galois connections [Cousot, 1992] is being studied. Such a holistic approach in considering the objectives of simulation explicitly into modeling via abstractions will help address the problem of validity and fidelity in simulation.

\section{CONCLUSIONS}

Primary EF component abstraction in input stimuli has been explained with respect to simulation objectives. The hierarchical abstraction for class of abstraction is explained with its correspondence to simulation objective. Validity is assessed with a behavioural compatibility criteria based on trace inclusion. The method implemented here is not correct by design but rather employed in classical iterative fashion which is clearly neither optimal nor formal in its approach. A rigorous mathematical framework in synthesising such an abstraction with respect to simulation objective would be the next step. However, the current study lays sufficient ground work in terms of assessment methodology for a formal abstraction compatibility criterion to be developed.

\section{ACKNOWLEDGEMENTS}

The authors would like to thank Richard Johnson and Bernard Mattos for reviewing the paper and Damien Foures for fruitful discussions on the landing gear example. 


\section{REFERENCES}

Albert, Vincent, 2009, Simulation validity assessment in the context of embedded system design, Phd Thesis, LAAS-CNRS, University of Toulouse, Unpublished.

Albert, V, Nketsa, A, Seguin, C, 2010, Verifying trace inclusion between an experimental frame and a model, DEVS Integrative Modeling and Simulation Symposium.

Boniol, F, Wiels, V, 2014, The Landing Gear Case Study, $4^{\text {th }}$ International ABZ Conference, Case study track.

Cousot, Patrick, 1992, Abstract Interpretation Frameworks, Journal of Logic and Computation, volume 2, pages 511-547.

Foures, D, Albert, V, Nkesta, A, 2013, Simulation validation using the compatibility between simulation model and experimental frame, Proceedings of the 2013 Summer Computer Simulation Conference, Society for Modeling \& Simulation International, Vista, CA, Article 55, 7 pages.

Foures, D, Albert, V, Nkesta, A, 2012, Formal compatibility of experimental frame concept and FDDEVS model, 9th International Conference on Modeling, Optimization \& Simulation, Bordeaux, France.

Girard, A, Pappas, G J, 2007, Approximation Metrics for Discrete and Continuous Systems, IEEE Transactions on Automatic Control, Volume 52, Issue 5, pages 782798.

Man-Kit-Leung, J, Mandl, T, Lee, E A, Latronico, E, Shelton, C, Tripakis, S, Lickly, B, 2009, Scalable semantic annotation using lattice based ontologies. Lecture Notes in Computer Science, Volume 5795, pp 393-407.

MATLAB SIMULINK Single hydraulic cylinder simulation, SIMULINK R2014a Example, http://www.mathworks.fr/fr/help/simulink/examples/si ngle-hydraulic-cylinder-simulation.html.

Traoré, M K, Muzzy, A, 2006, Capturing the dual relationship between simulation models and their context, Simulation Modelling Practice and Theory 14(2): 126-142.

Zeigler, B P, 1984, Theory of Modelling and Simulation, Krieger Publishing Co., Inc., Melbourne, FL, USA. 